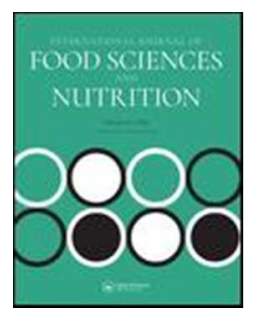

\title{
Teratogenic potential of nano-incapsulated vitamin A evaluated on an alternative model organism, the tunicate Ciona intestinalis.
}

\begin{tabular}{|r|l|}
\hline Journal: & International Journal of Food Sciences and Nutrition \\
\hline Manuscript ID & Draft \\
\hline Manuscript Type: & Research Paper \\
\hline Date Submitted by the Author: & n/a \\
\hline Complete List of Authors: & $\begin{array}{l}\text { Pennati, Roberta; Universita degli Studi di Milano, Department os } \\
\text { environmental scince and policy; } \\
\text { Manenti, Raoul; Universita degli Studi di Milano, Department of } \\
\text { environmental science and policy } \\
\text { Stillitano, Antonella; Universita degli Studi di Milano, Department of } \\
\text { environmental science and policy } \\
\text { Ficetola, Gentile; Universita degli Studi di Milano, Department os } \\
\text { environmental scince and policy } \\
\text { Scari, Giorgio; Universita degli Studi di Milano, Department of } \\
\text { environmental science and policy } \\
\text { Mercurio, Silvia; Università degli Studi di Milano, Department of } \\
\text { environmental science and policy } \\
\text { Menegola, Elena; Universita degli Studi di Milano }\end{array}$ \\
\hline Keywords: & \begin{tabular}{l} 
Functional food, Retinol, Bioavailability, ascidian \\
\hline
\end{tabular} \\
\hline
\end{tabular}

\section{SCHOLARONE \\ Manuscripts}




\title{
Teratogenic potential of nano-incapsulated vitamin A evaluated on an
} alternative model organism, the tunicate Ciona intestinalis.

\author{
Roberta Pennati ${ }^{1}$, Raoul Manenti ${ }^{1}$, Antonella Stillitano ${ }^{1}$, Gentile Francesco \\ Ficetola $^{1}$, Giorgio Scarì ${ }^{2}$, Silvia Mercurio ${ }^{1}$, Elena Menegola ${ }^{1}$ \\ ${ }^{1}$ Department of Environmental Science and Policy, University of Milano, Milano, Italy \\ ${ }^{2}$ Department of Biosciences, University of Milano, Milano, Italy
}

Corresponding author: Roberta Pennati, Department of Environmental Science and Policy, University of Milano, Via Celoria 2, 20133 Milano, Italy.

Phone: +390250314765

e-mail: roberta.pennati@unimi.it

Roberta Pennati was born in 1969 and graduated in Natural Sciences in 1993 at the University of Milano. She award a Ph.D. Degree in Natural and Environmental Sciences in 1998 at the University of Milano. Since 2002, she is research associate in Zoology at the Department of Biosciences, University of Milano. Her scientific activity concerns mainly developmental neurobiology of marine invertebrates with particular regard to tunicates.

\author{
Raoul Manenti: Department of Environmental Science and Policy, University of \\ Milano, Via Celoria 2, 20133 Milano, Italy. Phone:+393490733107 \\ e-mail: $\underline{\text { raoulmanenti@gmail.com }}$
}

Raoul Manenti graduated in Natural Sciences in at the University of Milano and Ph.D. in Animal Biology, he performs researches in different fields of the zoology, from developmental biology to herpetology. Currently he works as free-lance naturalist and collaborates with the Department of Environmental Science and Policy of the University of Milan. 
Antonella Stillitano: Via Maurizio Quadrio, 20 - 20154 Milano, Italy.

Phone: +39331321058

e-mail: antonstill@gmail.com

Antonella Stillitano graduated in Biology Applied to Sciences of Nutrition at the University of Milan, Department of Biosciences, Italy. She was internship at the Unit Clinic of Metabolic Diseases and Diabetes of A.O. S. Paolo, Milan, Italy. Currently she works as professional nutritionist.

Gentile Francesco Ficetola: Department of Environmental Science and Policy, University of Milano, Via Celoria 2, 20133 Milano, Italy.

Gentile Francesco Ficetola After a degree in natural sciences, Gentile Francesco Ficetola awarded a $\mathrm{PhD}$ in Environmental Sciences. He is a researcher combining genetic and statistical tools to understand the impact human activities and of environmental modifications on multiple levels of biodiversity.

e-mail: Francesco.ficetola@unimi.it

Giorgio Ulisse Scarì: Department of Biosciences, University of Milano, via Celoria 26, 20133 Milano, Italy. Phone: +39 0250314754

e-mail: giorgio.scari@unimi.it

Giorgio Ulisse Scarì: in1992 awarded the Ph.D. in ecology and evolutionary biology at the University of Milano. He is research associate at the Department of Biosciences, University of Milano. His research activity is mainly performed in the frame of Cell Biology, and its focused to the settling of synthesis methodologies, treatments and structural/functional characterizations of cells using nanocomposites and nanoparticles.

Silvia Mercurio: Department of Environmental Science and Policy, University of Milano, Via Celoria 2, 20133 Milano, Italy. +39 0250314755

e-mail: sil.mercurio@gmail.com 
Silvia Mercurio graduated in Biodiversity and Evolutionary Biology in 2010 at the University of Milano. She award a Ph.D. Degree in Animal Biology. Since 2014, she is postdoc fellow at the Department of Biosciences, University of Milano. Her scientific activity concerns developmental biology of invertebrate deuterostomes.

Elena Menegola: Department of Environmental Science and Policy, University of Milano, Via Celoria 2, 20133 Milano, Italy.

Elena Menegola graduated in Biological Science in 1990. She is Associate Professor of Comparative Anatomy at the University of Milano. Her main scientific interest concerns embryo development in alternative model animals to analyse the pathogenic pathways of congenital malformation following exposure to xenobiotics.

elena.menegola@unimi.it 


\title{
Teratogenic potential of nano-incapsulated vitamin A evaluated on an alternative model organism, the tunicate Ciona intestinalis.
}

\author{
Nano-encapsulation is a technology used to pack substances in order to enhance \\ stability and bioavailability that may interact with living systems causing \\ unexpected toxicity. Vitamin A is one of the substances that has received \\ attention, as in developed countries the increasing availability of supplements is \\ leading to its excessive intake. This study aims to compare teratogenic effects \\ caused by exposition to the traditional formulation of Vitamin A and nano- \\ encapsulated Vitamin A. We used ascidian embryos as alternative models. \\ Ascidians are marine organisms strictly related to vertebrates that share with \\ them the same body plan and developmental program, including the \\ morphogenetic role of retinoic acid (RA). Our data showed that adverse effects of \\ exposure to the same concentration of the two formulations were different \\ suggesting that the nano-encapsulation increased the bioavailability of the \\ molecule that could be better absorbed and metabolized to RA, the effective \\ teratogenic substance.
}

Keywords: functional food, retinol, ascidian, bioavailability.

\section{Introduction}

Nano-encapsulation is a novel technology used to pack substances in miniature. It is generally used to deliver different nutraceutical products and bioactive molecules such as vitamins and antioxidants, allowing production of functional foods with enhanced functionality and stability.

Lipid-based nano-encapsulation systems enhance the performance of lipophilic molecules by improving their solubility. In these systems, a lipophilic core is surrounded by an amphiphilic shell made of surface-active material that enhances solubility in aqueous media. Thus, nano-encapsulation can provide significant savings to formulators, as it allows to reduce the amount of active ingredients, increasing their bioavailability (Astete et al., 2009).

Moreover, several studies showed that the bioavailability of highly lipophilic substances encapsulated within lipid droplets increases as the droplet size decreases (Acosta, 2009). It has been proposed that the larger surface area of small droplets could allow quicker digestion leading to easier content release and absorption. In addition, small droplets 
have longer residence time in the small intestine and they can be directly transported across the epithelium by paracellular or transcellular mechanisms (McClements et al., 2011).

Besides, it is well known that nanoparticles furnish new chemical and physical properties, different from normal bulk formulation, and they may interact with living systems causing unexpected toxicity (Das et al., 2009). Indeed, there has been growing concern about the increased utilization of nanoparticles in foods and beverages because of potential toxic effects (Hagens et al., 2007; Chaudhry et al., 2008; Bouwmeester et al., 2009; Souto et al., 2009). Reducing the dimensions of a material to nanometer region may modify its biological fate within human body, such as absorption, distribution, metabolism, and excretion processes, thereby altering its potential for promoting toxicity (Hagens et al., 2007; Bouwmeester et al., 2009).

Since nanomaterials are essentially different from their corresponding bulk formulations, the European Food Safety Agency (EFSA) and the Food and Drug Administration (FDA) recommended to careful evaluate and monitor nano-formulated molecules since they can potentially cause risks to human health and environment (EFSA 2011).

On this regard, vitamin $\mathrm{A}$ is one of the vitamins which has received much attention as in developed countries the increasing availability of supplements is leading to excessive vitamin A intake (Penniston and Tanumihardjo, 2006), an even growing problem when considering nano-formulation diffusion.

Vitamin A (retinol) is mainly obtained from animal food. Once retinol has been taken up by a cell, it can be oxidized to retinal (retinaldehyde) by retinol dehydrogenases and then retinaldehyde can be oxidized to retinoic acid (RA) by aldehyde dehydrogenases (ALDHs).

RA is a morphogen known to play key roles during embryonic development of both vertebrate and invertebrate chordates (Canestro, 2006). RA levels are finely tuned in embryos by a precise balance between ALDH synthetic activity and the catalytic activity of CYP26, a cytochrome P450 enzyme. Embryos deprived of RA showed defects in different organs including eyes, heart, lungs and genital tract whereas early exposure to exogenous RA caused mainly defects in the anterior-posterior (AP) patterning of their neural tubes (Shimeld, 1998). Indeed, it has been demonstrated that in chordates RA regulates the patterning of the antero-posterior embryo body axis by controlling the expression of several HOX genes (Shimeld, 1998). 
Ascidians are chordates belonging to the Tunicate taxon that is considered the sister group of vertebrates (Delsuc et al., 2006). Adult ascidians are filter feeding sessile marine organisms; they develop through a swimming larva that retains the ancestral chordate body plan formed by a trunk and a locomotory tail where the notochord is located. A dorsal hollow neural tube constitutes the central nervous system; on the base of gene expression pattern, it can be subdivided in different regions homologue to forebrain, hindbrain and posterior neural tube of vertebrates (Passamaneck and Di Gregorio, 2005). Thus, ascidians, conjugating the handiness of an invertebrate with the body plan of a vertebrate, could represent an alternative model to investigate the toxicological potential of nano-molecules.

Exposure of ascidian gastrulae to exogenous RA causes a typical and highly reproducible phenotype characterized by round trunk (Nagatomo et al., 2003; De Bernardi et al., 1994). In the ascidian Ciona intestinalis, it has been demonstrated that exogenous RA upregulates $C i$-Cyp 26 expression and slightly downregulates $C i$-Aldh2 expression in the embryo (Nagatomo and Fujiwara, 2003). Moreover, RA strongly enhances Hox-1 expression both in C. intestinalis and in Halocynthia roretzi (Nagatomo and Fuijwara, 2003, Katsuyama et al., 1995).

In this work, we exposed ascidian embryos to nano-encapsulated and bulk vitamin A in order to compare the effects induced by the two formulations. We assumed that differences in the effects observed after treatment to the same concentrations could be attributed to the augmented bioavailability of the nano formulation thus obtaining an indirect assessment of its toxicity.

\section{Material and Method}

\section{Animals}

Adults of Ciona intestinalis were obtained by the fishery service of the Station Zoologique de Roscoff (France) and were maintained in aquaria filled with artificial seawater (Instant Ocean; salinity about 32\%o) and provided with internal circulation system as well as mechanical, chemical and biological filters. Constant illumination was preferred to prevent spontaneous release of gametes. Sperm and eggs were obtained surgically from animal gonoducts and used for in vitro fertilization in artificial sea water (ASWH: $122.75 \mathrm{~g} \mathrm{NaCl}, 3.35 \mathrm{~g} \mathrm{KCl} ; 7.35 \mathrm{~g} \mathrm{CaCl} 2$ 2H2O; 22.40g MgCl2 6H2O; 31.45g 
$\mathrm{MgSO}_{4} 7 \mathrm{H}_{2} \mathrm{O} ; 0.90 \mathrm{~g} \mathrm{NaHCO}_{3} ; 25 \mathrm{ml} 1 \mathrm{M}$ Hepes in 11 of $\mathrm{H}_{2} \mathrm{O}$ ), in order to obtain synchronously dividing embryos.

\section{Treatments}

Embryos at gastrula stage (4.9 hours post fertilization (hpf); Hotta et al., 2007) were exposed to different concentrations of retinol palmitate, the esterified and biological active form of vitamin A (Sigma, Italy), from here on bulk vitamin A (vit A), and to nanoencapsulated retinol palmitate (Aquanova ${ }^{\circledR}$ Novasol ${ }^{\circledR} \mathrm{GmbH}$, Germany), from here on nano vit $\mathrm{A}$.

Tested concentrations for both formulations were: $2 \mathrm{IU} / \mathrm{ml}, 10 \mathrm{IU} / \mathrm{ml}$ e $20 \mathrm{IU} / \mathrm{ml}$. Concentrations were chosen on the basis of previously reported effective concentrations of retinol on ascidian embryos (Groppelli et al., 2001).

Nano vit A is supplied as a $10 \%$ emulsion of nanoliposomes, formed by a shell of lipids from soya lecithin and a core of retinol palmitate. Stock solution of nano vit A was prepared diluting $11 \mu \mathrm{l}$ of emulsion in $20 \mathrm{ml}$ of $\mathrm{ASWH}$ and heating at $37^{\circ} \mathrm{C}$ for $15 \mathrm{~min}$ with gentle rocking. Working solutions were prepared diluting respectively $0.8,4$ and 8 $\mathrm{ml}$ of stock solution to a final volume of $40 \mathrm{ml}$ in ASWH.

$1 \mathrm{M}$ stock solution of bulk vit A corresponds to a $10.876 \mathrm{IU} / \mathrm{ml}$ concentration. Working solutions were prepared diluting respectively 736,368 and $73.6 \mu$ l of stock solution to a final volume of $40 \mathrm{ml}$ of ASWH.

C. intestinalis embryos at gastrula stage were treated in glass Petri dishes $(4 \mathrm{~cm}$ diameter) containing $40 \mathrm{ml}$ of working solution or $40 \mathrm{ml}$ of ASWH as control. An independent experiment was performed to test the effects of the nanoliposomes exposing embryos to the empty shells of soya lecithin (Aquanova ${ }^{\circledR}$ Novasol ${ }^{\circledR} \mathrm{GmbH}$, Germany). Each treatment was repeated 3 times on different batches. At least 50 embryos were exposed for each treatment. All treatments were performed at $18{ }^{\circ} \mathrm{C}$ in a thermostatic room. Embryos were allowed to develop until they reached the hatching larva stage (17.5 hpf; Hotta et al., 2007) and then fixed in 4\% paraformaldehyde for $1 \mathrm{~h}$ for following analysis.

\section{Morphological and statistical analysis}

Control and treated larvae were mounted on glasses for microscopic observations. Dead embryos and severely affected ones were counted. For the residual samples, the incidence of malformations at adhesive papillae, pigmented organs, trunk and tail was 
scored. We used generalized linear models to assess the impact of treatment and dose on larval mortality, and on the frequency of malformed individuals.

Subsequently, we repeated the analysis of the frequency of malformations on different portions of the body: adhesive papillae, pigmented organs, tail and trunk.

In all models, we used a quasi-binomial error distribution to take into account overdispersion, and we calculated significance using a F test (Maindonald and Braun, 2010). All analyses were performed using the $\mathrm{R}$ statistical environment (Maindonald and Braun, 2010).

A chi-square test was performed to disentangle differences between controls and embryos exposed to empty nanoliposomes. A $p$ value $\leq 0.05$ was considered significant.

\section{Immunostaining}

Larvae exposed to 10 and $20 \mathrm{IU} / 1$ of bulk and nano vit A were processed for immunostaining of nervous fibers with a monoclonal anti $\beta$ tubulin antibody (Sigma, clone 2-28-33).

Control and treated larvae were fixed in 4\% PFA in $0.1 \mathrm{M}$ phosphate buffer saline (PBS) at room temperature for $2 \mathrm{~h}$. All the following steps were performed with gentle rocking. Specimens were permeabilized with $0.1 \%$ Tween-20, $0.25 \%$ Triton X-100 in PBS for $1 \mathrm{~h}$, were washed three times in PBS for $10 \mathrm{~min}$ each, and incubated for $2 \mathrm{~h}$ in $50 \% \mathrm{PBS} / 50 \%$ normal goat serum. Then, the samples were incubated overnight at $4^{\circ} \mathrm{C}$ with primary antibody diluted 1:400. After several washes in PBS, the samples were incubated in $1 \%$ bovine serum albumin (BSA) in PBS for $2 \mathrm{~h}$ at room temperature, and then incubated at $4^{\circ} \mathrm{C}$ overnight in goat anti-mouse AlexaFluor 488 diluted 1:800 in PBS. After several washes in PBS, samples were mounted in 1,4diazabicyclo[2,2,2]octane (DABCO, Sigma, Italy) on microscope slides and examined using a confocal laser scanning microscope Leica TCSNT (Leica Microsystems, Heidelberg, Germany), equipped with laser argon/krypton, $75 \mathrm{~mW}$ multiline. Series of "optical sections" attained by scanning whole-mount specimens were projected into one image with greater focal depth.

\section{In situ hybridization}

Control and treated larvae were fixed in fresh $4 \%$ paraformaldehyde in $0.5 \mathrm{MNaCl}$ and $0.1 \mathrm{M}$ MOPS at $\mathrm{pH} 7.5$, at room temperature for $90 \mathrm{~min}$. Then, samples were washed twice with PBT $(0.1 \%$ Tween- $10 \%$ in PBS) and digested with $2 \mu \mathrm{g} / \mathrm{ml}$ Proteinase $\mathrm{K}$ for 
$30 \mathrm{~min}$ at $37^{\circ} \mathrm{C}$. Next, samples were post-fixed in $4 \%$ paraformaldehyde in PBS for $1 \mathrm{~h}$, washed twice in PBT and then three times, $10 \mathrm{~min}$ each, with $0.25 \%$ acetic anhydride in $0.1 \mathrm{M}$ triethanolamine. Samples were then incubated in the hybridization solution $(50 \%$ formamide, $5 \times \mathrm{SSC}, 50 \mu \mathrm{g} / \mathrm{ml}$ tRNA, $5 \times$ Denhardt's solution, $0.1 \%$ Tween- $10 \%$, $50 \mu \mathrm{g} / \mathrm{ml}$ heparin) for $1 \mathrm{~h}$. The hybridization was carried out overnight at $50^{\circ} \mathrm{C}$ with $0.3-$ $0.6 \mathrm{ng} / \mu \mathrm{l}$ Dig-labelled probe. $\mathrm{Ci}-\mathrm{Otx}$ and $\mathrm{Ci}-\mathrm{Hox}-1 \mathrm{dig}$ labelled antisense RNA was obtained by linearized plasmids using the dig-labelling kit (Roche, Italy) according to the indications. On the second day, samples were washed with a descendent series of SSC buffer in $50 \%$ formamide and $0.1 \%$ Tween- $10 \%$ then incubate overnight at $4{ }^{\circ} \mathrm{C}$ in a dilution (1:2000) of alkaline phosphatase-conjugated anti-DIG-antibody in blocking buffer containing $0.5 \%$ blocking reagent and 5\% normal sheep serum. On the third day, samples were washed several times in PBT and then rinsed with AP buffer containing NBT/BCIP substrates. When color reaction developed, larvae were washed in PBT, mounted on glass slides with $80 \%$ glycerol and observed using an optical microscope.

\section{Results}

\section{Morphological analysis}

Control larvae of Ciona intestinalis showed a normal phenotype characterized by an elongated trunk, pigmented organs correctly located in the sensory vesicle, three anterior palps well differentiated (Fig. 1A,B). Larvae exposed to low concentrations (2 $\mathrm{IU} / \mathrm{ml}$ ) of nano and bulk vit A showed a conserved morphology even if in some rare cases their palps were not elongated (Fig. 1C,F). Larvae exposed to the higher concentrations of vitamin A $(10 \mathrm{IU} / \mathrm{ml})$ nano and bulk showed recurrent malformations: palps were not correctly developed or were absent; pigmented organs were fused and dislocated in the upper side of the sensory vesicle; the trunk was roundish (Fig. 1D,G). Larvae exposed to the highest tested concentrations $(20 \mathrm{IU} / \mathrm{ml})$ of bulk vit A showed the same altered phenotypes (Fig. 1E). Instead, exposure to $20 \mathrm{IU} / \mathrm{ml}$ nano vit A caused a prevalent more severe phenotype characterized by a short trunk, short and curled tail, and absence of palps (Fig. 1H). Larvae exposed to the empty shell of soya lecithin were similar to control reared in ASWH (data not showed).

\section{Incidence of malformations}


Larvae developed from embryos exposed to Nano and Bulk Vitamin A were scored to obtain the proportion of not developed, healthy and malformed specimens. Resulting data were statistically analyzed to evaluate the effects of treatment, dose and the interaction of dose and treatment. Percentage of not developed larvae is not influenced by the treatment $(\mathrm{F} 1,14=0.52 ; \mathrm{P}=0.48)$, by the dose $(\mathrm{F} 2,14=1.01 ; \mathrm{P}=0.38)$ or by the interaction between dose and treatment $(\mathrm{F} 2,14=1.26 ; \mathrm{P}=0.31)$. These results prove that the two tested formulations of Vitamin A have no toxic effects on ascidian embryos and larvae.

Instead, statistical analysis showed a significant effect of dose $(\mathrm{F} 2,14=15.05 ; \mathrm{P}=$ $0.002)$ and of treatment $(\mathrm{F} 1,14=10.77 ; \mathrm{P}=0.005)$ on the incidence of malformed specimens, but not of the interaction between dose and treatment $(\mathrm{F} 2,14=1.26, \mathrm{P}=$ 0.31). The dose dependent incidence of malformations with both the formulations confirms that the teratogenic properties of Vitamin A are maintained following nano encapsulation. The most striking result is the significant effect of the two different formulations on the incidence of malformations, indicating that at the same dose the number of malformed specimens is higher after exposure to the nano encapsulated Vitamin A then to bulk Vitamin A. Figure 2A shows that exposure to $20 \mathrm{IU} / \mathrm{ml} \mathrm{Nano}$ Vitamin A causes more than $50 \%$ of malformed larvae $(53.4 \%)$, whereas the proportion of malformed larvae caused by the same dose of bulk Vitamin A is $11 \%$.

Larvae exposed to the empty shell of soya lecithin showed an incidence of malformed larvae very low and not significantly different from that of controls reared in ASWH (chi-square test: $\mathrm{P}=0.3105$ ). To better characterize the malformations induced, we identified four target organs and scored the exposed larvae according the presence of anomalies in these structures. From anterior to posterior these targets are: adhesive papillae, pigmented organs, trunk and tail. Data are reported in Table 1 and graphs in figure $2 \mathrm{~B}-\mathrm{E}$.

For the incidence of malformation to the adhesive papillae, there is a significant effect of the dose, but not of the treatment, that means that both the formulations of vit A have a similar dose dependent effect on the papillae, the anterior most organs. The incidence of malformations to the pigmented organs is significantly dependent by the dose and by the interaction between dose and treatment; in other words the differences between the two formulations are evident only at the highest tested doses. For the trunk malformations there are significant effects of concentrations and treatments. The interaction between concentration and treatment suggests that the differences between 
the two formulations are wider at the highest concentrations. Finally, there are no significant differences in the incidence of malformations of the tail, the more posterior organ of the larva.

\section{Immunostaining}

To better characterize induced malformations, we performed an immunostaining of the nervous system fibers of the larvae treated with the highest concentrations of nano and bulk vitamin A using an anti $\beta$-tubulin monoclonal antibody. In control larvae, the fibers of central and peripheral nervous system resulted well marked; in particular, papillary neurons and papillary nerves that run from the adhesive papillae to the posterior sensory vesicle were clearly recognizable (Fig. 3A). In the sensory vesicle, several fibers occurred in correspondence of the pigmented sensory organs, the otolith and the ocellus, as clearly visible in the image obtained by the superimpositions of confocal and transmission microscopy images (Fig. 3B). From the posterior part of the sensory vesicle, the neural tube fibers run posteriorly into the dorsal tail (Fig. 3A). The larvae exposed to $20 \mathrm{IU} / \mathrm{ml}$ bulk vit A showed the papillary nerves and neural fibers around the pigmented organs displaced more dorsally (Fig. 3C) as compared to control larvae (Fig. 3A); moreover the point of insertion of the sensory papillary nerves into the sensory vesicle was not clearly detectable. Fibers of the posterior neural tube were normally developed (Fig. 3C,D). Nervous fibers of the trunk of larvae exposed to 20 $\mathrm{IU} / \mathrm{ml}$ nano vit A were compromised severely. In particular, papillary nerves showed an abnormal pathway and were shorter than those of control larvae. Moreover, the fibers around pigmented organs were not detectable and the sensory vesicle was shifted anteriorly (Fig. 3E,F).

\section{In situ hybridization}

Defects to the sensory vesicle in exposed larvae were evidenced also by in situ hybridization experiments with a probe for $\mathrm{Ci}$-Otx. In Ciona intestinalis larvae, Ci-Otx is expressed exclusively in the sensory vesicle (Wada and Satoh, 2001) (Fig. 4A,B). In $20 \mathrm{IU} / \mathrm{ml}$ bulk vitamin A larvae, the region of expression of Ci-Otx was reduced indicating a reduction of the overall dimension of the sensory vesicle (Fig. 4C,D). In larvae exposed to $20 \mathrm{IU} / \mathrm{ml}$ nano vit A the sensory vesicle was slightly reduced as compared to larvae exposed to the bulk formulation and resulted displaced dorsally (Fig. 4E,F). 
To verify that the observed malformations were due to a specific effect of Vitamin A, we analyzed the expression of Ci-Hox-1 in control and exposed larvae. Ci-Hox-1 expression in greatly enhanced in Ciona intestinalis larvae by exposure to Retinoic Acid (RA), the active metabolite of Vitamin A (Nagatomo and Fujiwara, 2003). In control larvae, $\mathrm{Ci}$-Hox-1 was expressed in the endoderm of the trunk and in the neural tube, at level of visceral ganglion and in the first tract of the posterior nerve cord (Fig. 4G). In larvae exposed to $20 \mathrm{IU} / \mathrm{ml}$ bulk vit $\mathrm{A}$, the limit of expression of $\mathrm{Ci}-\mathrm{Hox}-\mathrm{l}$ in the neural tube was shifted posteriorly (Fig. $4 \mathrm{H}$ ). This shift was more marked in larvae exposed to the nano formulation of Vitamin A (Fig. 4I). These results suggested that the observed effects were attributable to the action of Vitamin A that upon its absorption is metabolized to RA.

\section{Discussion}

In this paper, we analyzed the effects that Vitamin A exposure on the development of $C$. intestinalis embryos. Moreover, we compared the effects induced by two different formulations of this molecule, bulk and nano-encapsulated, to evaluate if the latter can increase vitamin bio-availability.

Morphological analysis of exposed larvae indicated that vitamin A caused malformations similar to those observed after Retinoic Acid (RA) treatment principally affecting adhesive papillae, trunk and pigmented organs (Nagatomo et al., 2003; Katsuyama et al., 1995). Retinoic acid is the active metabolite of Vitamin A and is known to be a morphogen implicated in a wide range of biological processes during differentiation and morphogenesis including patterning of antero-posterior body axis (Maden and Holder, 1992). Generally, ascidian embryos exposure to RA results in a typical larval phenotype characterized by round trunk, reduced adhesive papillae, fusion of pigmented organs and extrusion of the sensory vesicle (Nagatomo et al., 2003).

In our experiments, we obtained the same phenotype, that was particularly accentuated in the samples exposed to the nano formulation.

Moreover, the incidence of malformations caused by exposure to vitamin A was dose and formulation dependent. Low concentrations of both formulations did not cause a significant increase of malformations as compared to controls. $2 \mathrm{IU} / \mathrm{ml}$ is the no observed effect level (NOEL) for both bulk and nano formulations of Vitamin A for $C$. intestinalis embryos. Whereas higher concentrations of nano-incapsulated Vitamin A 
induced a significant increment of severely malformed larvae as compared to the same concentration of the bulk form. We excluded the possibility that nanoliposomes are responsible of the observed malformations since treatments with empty shells did not caused any significant effect.

In addition, the severity of the induced malformations was dependent by the formulation. Immunolabelling of nervous fibers revealed that the anterior papillary nerves and the nervous fibers around the pigmented organs were more severely affected after exposure to the highest concentration of nano vit $\mathrm{A}$ than to the same concentration of the bulk form. The sensory vesicle, as shown by in situ hybridization with a Ci-Otx probe, was reduced and displaced dorsally following the exposure to nano vit A. This is a typical alteration caused by RA. The effects of exposure to the same concentration of the bulk form were less evident.

Considering all these aspects, our results suggested that the nano-encapsulation increased the bioavailability of the molecule that could be better absorbed and metabolized to RA. This observation is strengthened by the analysis of expression of Ci-Hox-1 in control and exposed larvae. In Ciona intestinalis larvae, Ci-Hox-1 is expressed with a sharp posterior limit in the nerve cord (Ikuta et al., 2005) and is inducible by exogenous RA (Nagatomo and Fujiwara, 2003). The increased expression of this gene in Vitamin A exposed larvae indicated that in these larvae the levels of RA augmented following an increment of the supply of its precursor. These data suggested that high concentrations of Vitamin A are teratogenic for ascidian larvae probably by inducing an increment of endogenous RA synthesis. Even though this seems to be the more plausible action of Vitamin A, it cannot be excluded that other mechanisms could be present, and the exact pathway of teratogenicity of this molecule need to be confirmed by further studies.

In any case, our data confirm that nano-incapsulated vit A differs in its effects to bulk formulation at the same doses and can potentially be a risk to human health.

In the last decade, much attention has been paid to project delivery systems to increase the bioavailability of approved food grade nutrients and bioactive substances, in order to produce functional foods or fortified foods, requested by consumers. Food products are normally fortified with health promoting and disease preventing molecules such as phytochemicals, vitamins, minerals, oils (omega 3 fatty acids) (Adytia et al., 2017).

In developed nations, due to fortified food, observational studies suggest that more than $75 \%$ of people may be routinely ingesting more than the recommended dietary 
allowance (RDA) for vitamin A (Allen and Haskell, 2002). The use of nanoencapsulated vit A can increase the risk of hyper-vitaminosis in these situations. Thus, our results suggest that it is necessary to carefully monitor beverage and food supplementation with nano-vit A and reconsider the RDA in the light of its augmented bioavailability, in accordance with recommendations of European Food Safety Agency (EFSA) and the Food and Drug Administration (FDA).

Acknowledgments: The authors want to thank the people of "IBIS: imaging bioscience support" for the use of confocal microscope.

Disclosure of interest: the authors report no conflicts of interest.

\section{References}

Acosta E. 2009. Bioavailability of nanoparticles in nutrient and nutraceutical delivery. J. Colloid Interface Sci 14: 3-15.

Aditya NP, Espinosa YG, Norton IT. 2017. Encapsulation systems for the delivery of hydrophilic nutraceuticals: food application. Biotechnology Advances

Allen LH, Haskell M. 2002. Estimating the potential for vitamin A toxicity in women and young children. J Nutr.132:2907S-19S.

Astete CE, Sabliov CM, Watanabe F, Biris A. 2009. Ca2+ cross-linked alginic acid nanoparticles for solubilization of lipophilic natural colorants. J Agric Food Chem. $57: 7505-7512$.

Bouwmeester H, Dekkers S, Noordam MY, Hagens WI, Bulder AS, de Heer C, ten Voorde SECG, Wijnhoven SWP, Marvin HJP, SipsAJAM. 2009. Review of health safety aspects of nanotechnologies in food production. Review of health safety aspects of nanotechnologies in food production, Regulatory Toxicology and Pharmacology. 53:52-62. 
Canestro C, Postlethwait JH, Gonzalez-Duarte R, Albalat R. 2006. Is retinoic acid genetic machinery a chordate innovation? Evol. Dev. 8:394 -406

Chaudhry Q, Scotter M, Blackburn J, Ross B,Boxall A, Castle L, Aitken R, Watkins R. 2008. Applications and implications ofnanotechnologies for the food sector, Food Additives \& Contaminants: Part A, 25:3, 241-258, DOI:10.1080/02652030701744538

Das M, Saxena N, Dwivedi PD. 2009. Emerging trends of nanoparticles application in food technology: Safety paradigms. Nanotoxicology. 3:10-18.

De Bernardi F, Sotgia C, Ortolani G. 1994. Retinoic acid treatment of ascidian embryos: Effects on larvae and metamorphosis. Anim Biol 3: 75-81.

Delsuc F, Brinkmann H, Chourrout D, Philippe H., 2006. Tunicates and not cephalochordates are the closest living relatives of vertebrates. Nature. 439, 965-968.

EFSA Scientific Committee. 2011. Guidance on the risk assessment of the application of nanoscience and nanotechnology in the food and feed chain. EFSA Journal 9: 2140.

Groppelli S, Pennati R, Sotgia C, De Bernardi F. 2001. AChE localization in the adhesive papillae of the ascidian larvae: effects of citral, a retinoic acid synthesis inhibitor. Invertebr Reprod Dev. 40: 95-102.

Hagens WI, Oomen AG, de Jong WH, Cassee FR, Sips AJ. 2007. What do we (need to) know about the kinetic properties of nanoparticles in the body?Regul Toxicol Pharmacol. 49:217-229.

Hotta K, Mitsuhara K, Takahashi H, Inaba K, Oka K, Gojoboti T, Ikeo K. 2007. A web-based interactive developmental table for the ascidian Ciona intestinalis, including 3D real-image embryo reconstructions: I. From fertilized egg to hatching larva. Dev Dyn 236:1790-805.

Ikuta T and Saiga H. 2005. Organization of Hox genes in ascidians: present, past and future. Dev Dyn 233:382-389. 
Katsuyama Y, Wada S, Yasugi S, Saiga H, 1995. Expression of the labial group Hox gene HrHox-1 and its alteration induced by retinoic acid in development of the ascidian Halocynthia roretzi. Development 121: 3197-3205.

Maden M, Holder N. 1992.Retinoic acid and development of the central nervous system. Bioessays 14:431-438.

Maindonald, J., Braun, W.J., 2010. Data analysis and graphics using R. Cambridge University Press, Cambridge.

McClements DJ, Rao J. 2011. Food-grade nanoemulsion: formulation, fabrication, properties, performance, biological fate and potential toxicity. Crit Rew Food Sci. 51: 285-330.

Nagatomo K, Ishibashi T, Satou Y, Satoh N, Fujiwara S. 2003. Retinoic acid effects gene expression and morphogenesis without upregulating the retinoid acid receptor in the ascidian Ciona intestinalis. Mech Dev. 120: 363-372.

Nagatomo K, Fujiwara S. 2003. Expression of Raldh2, Cyp26 and Hox-1 in normal and retinoic acid-treated Ciona intestinalis embryos. Gene Expr Patterns. 3:273-277.

Passamaneck YJ, Di Gregorio A. 2005. Ciona intestinalis: chordate development made simple. Dev Dyn. 233:1-19.

Penniston KL, Tanumihardjo SA. 2006. The acute and chronic toxic effects of vitamin A. Am J Clin Nutr 83:191-201.

Souto EB, Martins-Lopes P, Lopes CM, Gaivão I, Silva AM, Guedes-Pinto H. 2009.A note on regulatory concerns and toxicity assessment in lipid-based delivery systems (LDS).J Biomed Nanotechnol. 5:317-322.

Wada H, Satoh N. 2001.Patterning the protochordate neural tube. Curr Opin Neurobiol $11: 16-21$. 
Figure 1: Typical phenotypes obtained after exposure to bulk and nano vit A. A Control larva. B. Schematic drawing of the anatomy of a larva, main organs are indicated with a color code. C-E: larvae exposed to bulk vit A. F-H: larvae exposed to Nano- vitamin A. Scale bar: $100 \mu \mathrm{m}$.

Figure 2: Graphs indicating the incidence of severely malformed larvae (A) and the incidence of the malformations at target organs (B-D) after exposure to different concentrations of bulk and nano vit $\mathrm{A}$. The values are in percentage and standard deviation is indicated.

Figure 3: Confocal laser microscope images of the trunk of the larvae. Nervous fibers are immunolabelled with anti- $\beta$ tubulin antibody. A,B: Control larva. C,D: larva exposed to $20 \mathrm{IU} / \mathrm{ml}$ bulk vit A. E,F: larva exposed to $20 \mathrm{IU} / \mathrm{ml}$ nano vit A. B,D,E: superimposition of confocal microscope image with a transmission microscope one. ap, adhesive papilla; nt, neural tube; oc, ocellus; ot, otolith; pn, papillary neurons; sv, sensory vesicle.

Figure 4: In situ hybridizations. A-F: Ci-Otx expression. A,B: control larva. C,D: larva exposed to $20 \mathrm{IU} / \mathrm{ml}$ bulk vitamin A. E,F: larva exposed to $20 \mathrm{IU} / \mathrm{ml}$ nano vitamin A. GI: expression of $\mathrm{Ci}-\mathrm{Hox}-\mathrm{l}$. The arrow indicates the posterior limit of expression. G: control larva. H: larva exposed to $20 \mathrm{IU} / \mathrm{ml}$ bulk vitamin A. I: larva exposed to $20 \mathrm{IU} / \mathrm{ml}$ nano vitamin A. Scale bar. $100 \mu \mathrm{m}$.

Table 1: Effect of dose and treatment on the frequency of malformations in the different body portions. Significant effects are in bold. 
Figure 1: Typical phenotypes obtained after exposure to bulk and nano vit A. A Control larva. B. Schematic drawing of the anatomy of a larva, main organs are indicated with a color code. C-E: larvae exposed to bulk vit A. F-H: larvae exposed to Nano- vitamin A. Scale bar: $100 \mu \mathrm{m}$.

$$
164 \times 57 \mathrm{~mm}(300 \times 300 \mathrm{DPI})
$$



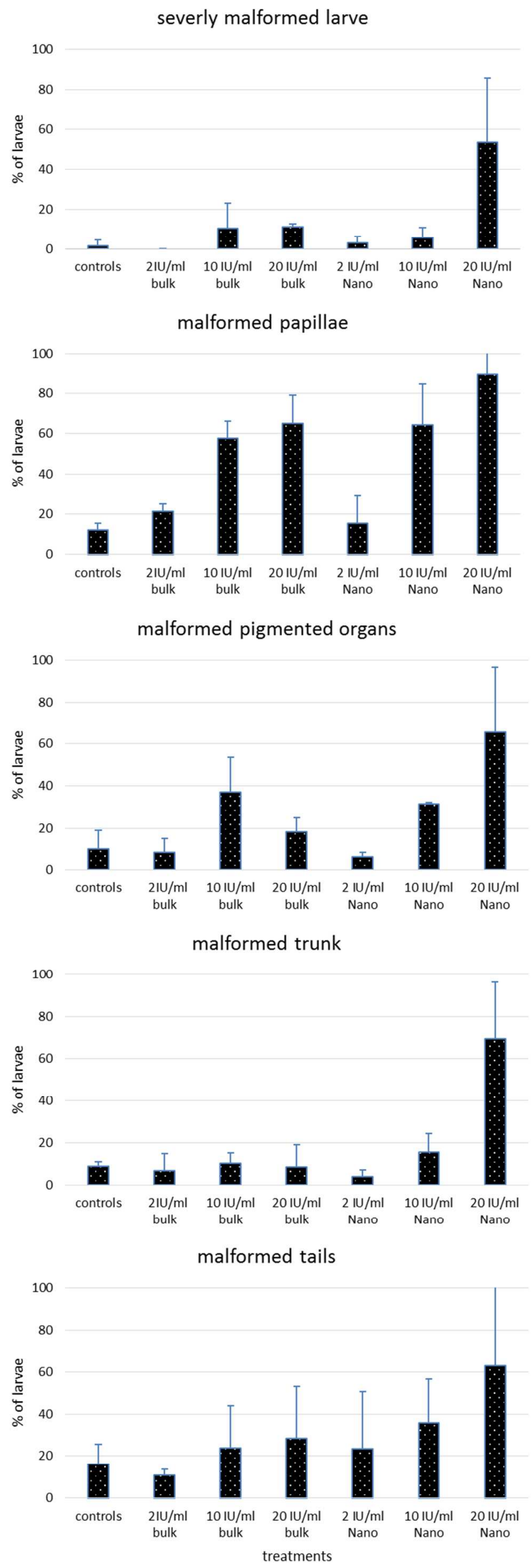

URL: http://mc.manuscriptcentral.com/cijf Email: daniele.delrio@unipr.it 

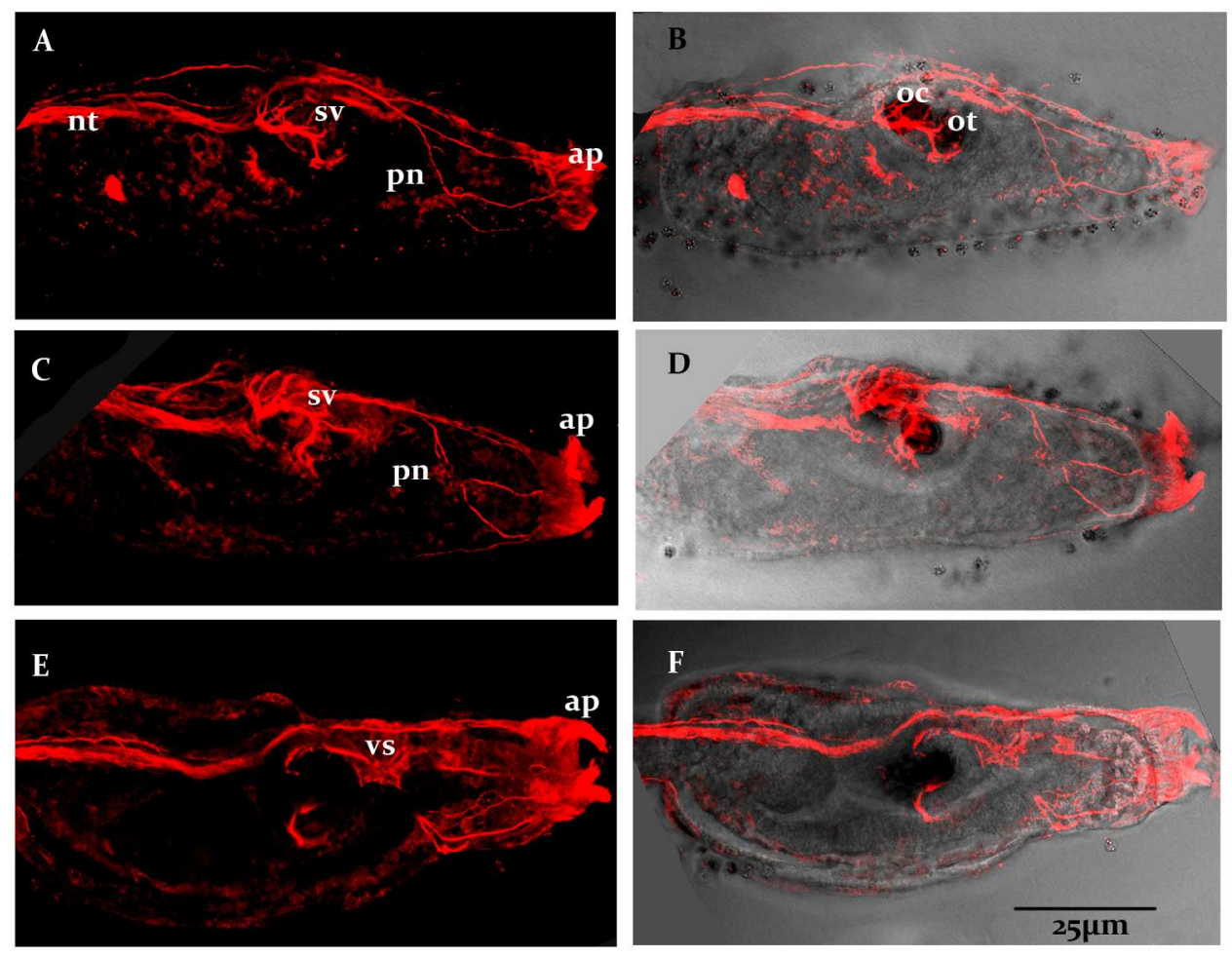

Figure 3: Confocal laser microscope images of the trunk of the larvae. Nervous fibers are immunolabelled with anti- $\beta$ tubulin antibody. A,B: Control larva. C,D: larva exposed to $20 \mathrm{IU} / \mathrm{ml}$ bulk vit $\mathrm{A}$. E, F: larva exposed to $20 \mathrm{IU} / \mathrm{ml}$ nano vit A. B,D,E: superimposition of confocal microscope image with a transmission microscope one. ap, adhesive papilla; nt, neural tube; oc, ocellus; ot, otolith; pn, papillary neurons; sv, sensory vesicle.

$200 \times 156 \mathrm{~mm}(300 \times 300 \mathrm{DPI})$ 


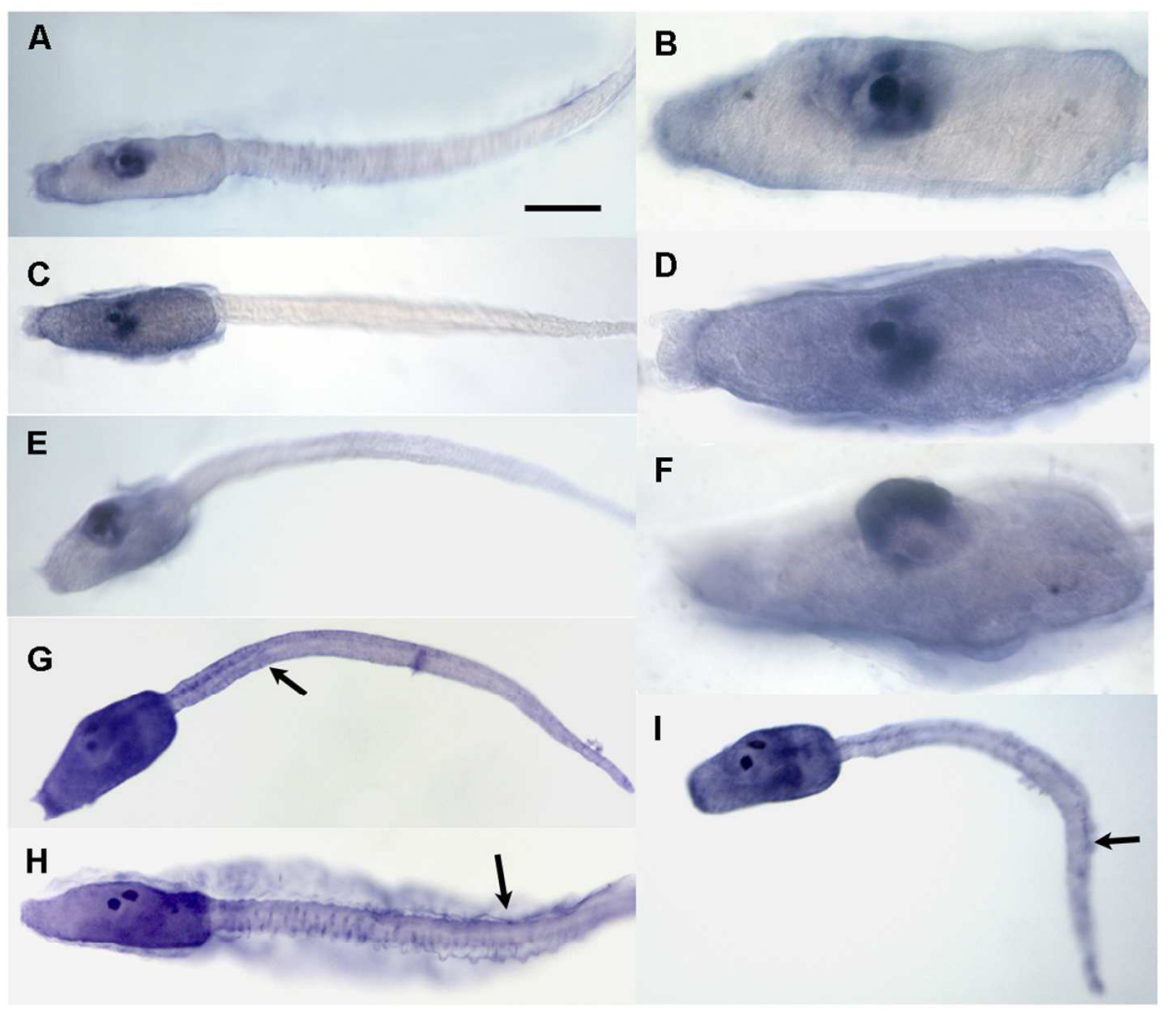

Figure 4: In situ hybridizations. A-F: Ci-Otx expression. A,B: control larva. C,D: larva exposed to 20IU/ml bulk vitamin A. E,F: larva exposed to 20IU/ml nano vitamin A. G-I: expression of Ci-Hox-1. The arrow indicates the posterior limit of expression. G: control larva. H: larva exposed to $20 \mathrm{IU} / \mathrm{ml}$ bulk vitamin A. I: larva exposed to $20 \mathrm{IU} / \mathrm{ml}$ nano vitamin A. Scale bar. $100 \mu \mathrm{m}$.

$$
106 \times 93 \mathrm{~mm}(300 \times 300 \text { DPI })
$$




\begin{tabular}{|l|l|l|l|l|l|l|l|l|l|}
\hline & \multicolumn{4}{|l}{ concentration } & \multicolumn{3}{l}{ treatment } & \multicolumn{3}{l|}{$\begin{array}{l}\text { concentration } \times \\
\text { treatment }\end{array}$} \\
\hline & $F$ & d.f. & $P$ & $F$ & d.f. & $P$ & $F$ & d.f. & $P$ \\
\hline Adhesive papillae & 11.08 & 2,14 & $\mathbf{0 . 0 0 1}$ & 0.18 & 1,14 & 0.67 & 1.61 & 2,14 & 0.24 \\
\hline Pigmented organs & 8.43 & 2,14 & $\mathbf{0 . 0 0 4}$ & 1.82 & 1,14 & 0.20 & 5.24 & 2,14 & $\mathbf{0 . 0 2}$ \\
\hline Trunk & 9.12 & 2,14 & $\mathbf{0 . 0 0 3}$ & 12.15 & 1,14 & $\mathbf{0 . 0 0 4}$ & 4.96 & 2,14 & $\mathbf{0 . 0 2}$ \\
\hline Tail & 1.14 & 2,14 & 0.35 & 1.66 & 1,14 & 0.22 & 0.21 & 2,14 & 0.81 \\
\hline
\end{tabular}


A

\begin{tabular}{|c|c|c|c|c|c|c|c|}
\cline { 2 - 7 } & controls & 2 IU/mI bulk & 10 IU/mI bulk & 20 IU/mI bulk & 2 IU/mI Nano & 10 IU/mI Nano 20 IU/mI Nano \\
\hline $\begin{array}{c}\text { examined } \\
\text { larvae }\end{array}$ & 201 & 158 & 174 & 150 & 157 & 167 & 155 \\
\hline $\begin{array}{c}\text { not } \\
\text { developed }\end{array}$ & $6(2.99 \%)$ & $19(12.03 \%)$ & $9(5.17 \%)$ & $15(10.00 \%)$ & $14(8.92 \%)$ & $17(10.18 \%)$ & $20(12.90 \%)$ \\
\hline $\begin{array}{c}\text { severely } \\
\text { malf. larvae }\end{array}$ & $5(2.49 \%)$ & 0 & $8(4.60 \%)$ & $17(11.33 \%)$ & $5(3.18 \%)$ & $8(4.79 \%)$ & $81(52.26 \%)$ \\
\hline $\begin{array}{c}\text { healthy } \\
\text { larvae }\end{array}$ & $190(94.53 \%)$ & $139(87.97 \%)$ & $157(90.23 \%)$ & $118(78.67 \%)$ & $138(87.90 \%)$ & $142(85.03 \%)$ & $54(34.84 \%)$ \\
\hline
\end{tabular}

B

\begin{tabular}{|c|c|c|c|c|c|c|c|}
\cline { 2 - 7 } & controls & 2IU/mI bulk & 10 IU/mI bulk & 20 IU/mI bulk & 2 IU/mI Nano & 10 IU/mI Nano & 20 IU/mI Nano \\
\hline $\begin{array}{c}\text { examined } \\
\text { larvae }\end{array}$ & 116 & 115 & 140 & 125 & 99 & 74 & 87 \\
\hline $\begin{array}{c}\text { malformed } \\
\text { palps }\end{array}$ & $14(12.07 \%)$ & $25(21.74 \%)$ & $81(57.86 \%)$ & $81(64.80 \%)$ & $16(16.16 \%)$ & $51(68.925)$ & $79(90.80 \%)$ \\
\hline $\begin{array}{c}\text { malformed } \\
\text { pig. organs }\end{array}$ & $13(11.21 \%)$ & $9(7.83 \%)$ & $51(36.43 \%)$ & $23(18.40 \%)$ & $6(6.06 \%)$ & $23(31.08 \%)$ & $55(63.22 \%)$ \\
\hline $\begin{array}{c}\text { malformed } \\
\text { trunk }\end{array}$ & $11(9.485)$ & $8(6.96 \%)$ & $14(10.00 \%)$ & $11(8.80 \%)$ & $4(4.04 \%)$ & $13(17.57 \%)$ & $59(67.82 \%)$ \\
\hline $\begin{array}{c}\text { malformed } \\
\text { tail }\end{array}$ & $21(18.10 \%)$ & $12(10.43 \%)$ & $34(24.29 \%)$ & $35(28.00 \%)$ & $25(25.25 \%)$ & $30(40.54 \%)$ & $51(58.62 \%)$ \\
\hline
\end{tabular}

C

\begin{tabular}{|c|c|c|}
\cline { 2 - 3 } \multicolumn{1}{c|}{} & controls & $\begin{array}{c}\text { empty nano- } \\
\text { liposomes }\end{array}$ \\
\hline $\begin{array}{c}\text { examined } \\
\text { larvae }\end{array}$ & 160 & 170 \\
\hline $\begin{array}{c}\text { not } \\
\text { developed }\end{array}$ & $18(11.25 \% \%)$ & $12(7.05 \%)$ \\
\hline $\begin{array}{c}\text { severely } \\
\text { malf. larvae }\end{array}$ & $17(10,60 \%)$ & $11(6.47 \%)$ \\
\hline $\begin{array}{c}\text { healthy } \\
\text { larvae }\end{array}$ & $125(78.12 \%)$ & $147(86.47 \%)$ \\
\hline
\end{tabular}

